\title{
FAMILIES OF SOLUTIONS OF A PERTURBATION PROBLEM
}

\section{JANE CRONIN}

1. Introduction. In previous papers $[2 ; 3]$, topological degree theory was used to establish the existence of periodic solutions of systems of ordinary differential equations with a nonlinear perturbation term. In $[2 ; 3]$, methods for computing the appropriate topological degree were developed and in [3], it was shown that for 2-dimensional, totally degenerate systems, the absolute value of the topological degree, call it $|d|$, is a lower bound for the number of distinct periodic solutions in the following sense: if the perturbation term in the system of differential equations contains a term which is a function of the independent variable only (such a term is often called a forcing term) then if this term is varied arbitrarily slightly, the number of distinct periodic solutions is greater than or equal to $|d|$.

In this note, we extend and sharpen this result. First we show that the result holds for systems of arbitrary dimension and with arbitrary degree of degeneracy. Secondly, we show that these periodic solutions form families continuous in the perturbation parameter.

As in [3], we start from the treatment of perturbed systems given by Coddington and Levinson [1, pp. $356 \mathrm{ff}$.]. This note is independent of $[2 ; 3]$ and may be regarded as an extension of the theory developed in $[1$, pp. $356 \mathrm{ff}$. $]$.

2. Families of solutions. We consider the $n$-dimensional system,

$$
\dot{x}=A x+\mu f(x, t, \mu),
$$

where $A$ is a constant matrix, $\mu$ is a parameter, and $f$ has period $2 \pi$ in variable $t$. We study the degenerate case, i.e., the case in which the linear part of (E) [the equation $\dot{x}=A x$ ] has $q$ nonzero solutions of period $2 \pi$ where $1 \leqq q \leqq n$. We assume that $f$ has continuous second derivatives in all values of $x, t, \mu$. Let $x(t, \mu, c)$ be the general solution of (E) such that $x(0, \mu, c)=c$. Because of the uniqueness condition in the general existence theorem, the condition that $x(t, \mu, c)$ have period $2 \pi$ is:

$$
x(2 \pi, \mu, c)-c=0,
$$

or using the variation of constants formula,

Presented to the Society, February 27, 1960; received by the editors November 23, 1959; and, in revised form, February 18, 1960. 


$$
\left(e^{2 \pi A}-E\right) c+\mu \int_{0}^{2 \pi} e^{(2 \pi-s) A} f[x(s, \mu, c), s, \mu] d s=0,
$$

where $E$ is the identity matrix. (Equation (1) is Equation (3.20) on p. 360 in Coddington and Levinson [1].) Thus the problem of finding periodic solutions of $(\mathrm{E})$ is that of solving the system (1) of $n$ equations for the $n$ unknowns, $c_{1}, \cdots, c_{n}$, the components of $c$.

Following [1] we make the following assumption.

Assumption 1. Matrix $A$ has the canonical form:

$$
A=\left(\begin{array}{lllllll}
A_{1} & & & & & \\
& \ddots & & & & \\
& & A_{k} & & & \\
& & B_{1} & & \\
& & & \ddots & & \\
& & & & B_{m} & \\
& & & & & C
\end{array}\right)
$$

where the elements not shown are zeros. Each $A_{j}, j=1, \cdots, k$, is a matrix of $\alpha_{j}$ ( $\alpha_{j}$ even) rows and columns of the form

$$
A_{j}=\left(\begin{array}{llll}
S_{j} & & & \\
E_{2} & S_{j} & & \\
& & \ddots & \\
& & E_{2} S_{j}
\end{array}\right)
$$

where all the elements are zero except $S_{j}$ and $E_{2}$, and

$$
S_{j}=\left(\begin{array}{cc}
0 & -N_{j} \\
N_{j} & 0
\end{array}\right), \quad E_{2}=\left(\begin{array}{ll}
1 & 0 \\
0 & 1
\end{array}\right)
$$

where $N_{j}$ is a positive integer. (A matrix $A_{j}$ may have only two rows and two columns in which case $A_{j}=S_{j}$.) Each matrix $B_{j}$ has $\beta_{j}$ rows and columns, $j=1, \cdots, m$, and is of the form

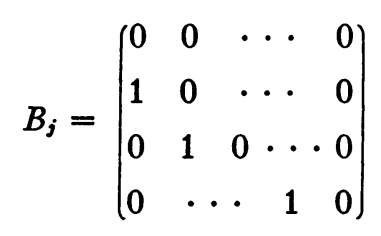

where $B_{j}$ may have only one row and column in which case $B_{j}$ consists of the single element 0 . The matrix $C$ has $\left(n-\sum_{j=1}^{k} \alpha_{j}-\sum_{j=1}^{m} \beta_{j}\right)$ 
rows and columns and has no characteristic roots of the form $i N$ for any integer $N$ including $N=0$. Matrix $C$ need not be in canonical form.

If $\left(c_{1}, \cdots, c_{i}, \cdots, c_{n}\right)$ is an $n$-vector, the indices $i$ corresponding to the last two rows of any $A_{j}$ or to the last row of any $B_{j}$ are called exceptional indices. They are indices with the following form:

$$
\begin{aligned}
& i=\alpha_{1}+\alpha_{2}+\cdots+\left(\alpha_{j}-1\right), \\
& i=\alpha_{1}+\alpha_{2}+\cdots+\alpha_{j},
\end{aligned}
$$

where $j=1, \cdots, k$, and

$$
i=\alpha_{1}+\cdots+\alpha_{k}+\beta_{1}+\cdots+\beta_{j}
$$

where $j=1, \cdots, m$. The indices $i$ corresponding to the first two rows of any $A_{j}$ or to the first row of any $B_{j}$ are called singular indices. They are indices with the following form:

$$
\begin{aligned}
i= & 1,2, \alpha_{1}+1, \alpha_{2}+2, \cdots, \alpha_{1}+\alpha_{2}+\cdots+\alpha_{k-1}+1, \\
& \alpha_{1}+\alpha_{2}+\cdots+\alpha_{k-1}+2, \alpha_{1}+\cdots+\alpha_{k}+1, \\
& \alpha_{1}+\cdots+\alpha_{k}+\beta_{1}+1, \cdots, \alpha_{1}+\cdots+\alpha_{k}+\beta_{1}+\cdots \\
& +\cdots \\
& +\beta_{m-1}+1 .
\end{aligned}
$$

There are $(2 k+m)$ exceptional indices and $(2 k+m)$ singular indices. The number $q=2 k+m$ is the degree of degeneracy of the problem. Throughout this paper, we assume that $q>0$, i.e., that there is at least one $A_{j}$ or one $B_{j}$ in the canonical form of matrix $A$.

As described in [1], if the $c_{1}, \cdots, c_{n}$ are relabelled $c_{1}^{\prime}, \cdots, c_{n}^{\prime}$ so that the first $(n-q) c_{i}^{\prime}$ 's are the components of $\left(c_{1}, \cdots, c_{n}\right)$ with nonexceptional indices and the last $q c_{i}^{\prime}$ 's are the components with exceptional indices and if $j, j^{\prime}$ denote singular and nonsingular indices, then equation (1) may be replaced with:

$$
\begin{aligned}
N\left(c_{1}^{\prime}, \cdots, c_{n-q}^{\prime}\right)+\mu & \left(\int_{0}^{2 \pi} e^{(2 \pi-8) A} f[x(s, \mu, c), s, \mu] d s\right)_{\left(j^{\prime}\right)}=0, \\
& \left(\int_{0}^{2 \pi} e^{(2 \pi-8) A} f[x(s, \mu, c), s, \mu] d s\right)_{(j)}=0,
\end{aligned}
$$

where $N$ is a nonsingular $(n-q) \times(n-q)$ matrix acting on vector $\left(c_{1}^{\prime}, \cdots, c_{n-q}^{\prime}\right)$ and $\left(\int_{0}^{2 \pi} e^{(2 \pi-s) A} f[x(s, \mu, c) s, \mu] d s\right)_{j^{\prime}}$ denotes the vector composed of the $(n-q)$ components of $\int_{0}^{2 \pi} e^{(2 \pi-s)} A f[x(s, \mu, c), s, \mu] d s$ which have nonsingular indices. Similarly

$$
\left(\int_{0}^{2 \pi} e^{(2 \pi-s) A} f[x(s, \mu, c), s, \mu] d s\right)_{(j)}
$$


denotes the vector composed of the $q$ components which have singular indices.

The left side of (2) defines a continuous mapping (call it $\mathfrak{T}_{\mu}$ ) of real Euclidean $n$-space into itself. Let $c^{\prime \prime}$ denote the vector $c$ in which the $(n-q)$ components with nonexceptional indices have been set equal to zero. (The nonzero components of $c^{\prime \prime}$ are $c_{n-q+1}^{\prime}, \cdots, c_{n}^{\prime}$.) Then

$$
\left(\int_{0}^{2 \pi} e^{(2 \pi-8) A} f\left[e^{s A} c^{\prime \prime}, s, 0\right] d s\right)_{(j)}
$$

defines a continuous mapping of real $q$-space into itself which we call $M$.

Lemma 1. If $\left(c_{n-q+1}^{0}, \cdots, c_{n}^{0}\right)$ is a solution of

$$
M\left(c_{n-q+1}^{\prime}, \cdots, c_{n}^{\prime}\right)=0
$$

at which the Jacobian of $M$ (denote it by $J(M)$ ) is $\neq 0[=0]$, then $\left(0, \cdots, 0, c_{n-q+1}^{0}, \cdots, c_{n}^{0}\right)$ is a solution of

$$
\operatorname{Tr}_{0}\left(c_{1}^{\prime}, \cdots, c_{n}^{\prime}\right)=0
$$

at which $J\left(M_{0}\right) \neq 0[=0]$. Conversely any solution of (4) has the form $\left(0, \cdots, 0, c_{n-q+1}^{0}, \cdots, c_{n}^{0}\right)$ where $\left(c_{n-q+1}^{0}, \cdots, c_{n}^{0}\right)$ is a solution of $(3)$, and if $J\left(M_{0}\right) \neq 0[=0]$ at $\left(0, \cdots, 0, c_{n-a+1}^{0}, \cdots, c_{n}^{0}\right)$, then $J(M)$ $\neq 0[=0]$ at $\left(c_{n-q+1}^{0}, \cdots, c_{n}^{0}\right)$.

Proof. Follows from inspection of systems (3) and (4).

Lemma 2. Let $S$ be a sphere in $n$-space with center at the origin and radius $r$, and let $S$ be a sphere in q-space with center at the origin and radius $r$. If the topological degree of $M$ at $O$ (the origin in q-space) and relative to $S$ exists, then if $\mu$ is sufficiently small, the topological degree of $\mathfrak{M} \mu$ at $O$ (the origin of the $n$-space) and relative to $S$ exists and equals the topological degree of $M$.

Proof. Follows from the definition of topological degree and the fact that the topological degree is invariant under homotopy.

Now we make the following assumption:

Assumption 2. Let $f_{1}(x, t, \mu), \cdots, f_{n}(x, t, \mu)$ denote the components of $f(x, t, \mu)$. We assume that if $j$ is a singular index, then

$$
f_{j}(x, t, \mu)=g_{j}(x, t, \mu)+h_{j}(t)
$$

where $h_{j}(t)$ is a function of $t$ only with a continuous second derivative and $h_{j}(t)$ has period $2 \pi$ in $t$.

Now let $h(t)$ be the vector whose $j$ th component is 0 if $j$ is nonsingular and whose $j$ th component is $h_{j}(t)$ if $j$ is singular. Similarly 
define the vector $g(t)$. If Assumption 2 is satisfied, then system (4) has the form:

$$
\begin{aligned}
N\left(c_{1}^{\prime}, \cdots, c_{n-q}^{\prime}\right) & =0 \\
\left(\int_{0}^{2 \pi} e^{(2 \pi-s) A} g\left[e^{s A} c, s, 0\right] d s\right)_{(j)} & =-\left(\int_{0}^{2 \pi} e^{(2 \pi-s) A} h(s) d s\right)_{(j)},
\end{aligned}
$$

where $j$ denotes a singular index. We want to write the constant terms on the right in (5) in detail. First, these terms may be written:

$$
-\left(\int_{0}^{2 \pi} e^{s A} h(-s) d s\right)_{(j)}
$$

From the definition of singular index, the terms may then be written:

$$
\begin{gathered}
-\int_{0}^{2 \pi}\left[\cos \left(N_{1} s\right) h_{1}(-s)-\sin \left(N_{1} s\right) h_{2}(-s)\right] d s=b_{1} \\
-\int_{0}^{2 \pi}\left[\sin \left(N_{1} s\right) h_{1}(-s)+\cos \left(N_{1} s\right) h_{2}(-s)\right] d s=b_{2} \\
\ldots \ldots \ldots \ldots \ldots \ldots \ldots \ldots \\
-\int_{0}^{2 \pi}\left[h_{q}(-s)\right] d s=b_{q} .
\end{gathered}
$$

The form of the last equation in system (6) implies that there is a $B_{j}$ in the canonical form of matrix $A$. If there is no $B_{j}$ in the canonical form, then all the equations in system (6) look like the first two equations in the system.

Now the point $b=\left(b_{1}, \cdots, b_{q}\right)$ is a point in $q$-space.

Lemma 3. Given $\epsilon_{0}>0$, an arbitrary positive number, then there exists $a$ neighborhood $N$ of $b$ in $q$-space such that if $p \in N$, then there exist $h_{1}^{(1)}(t), \cdots, h_{a}^{(1)}(t)$, all of period $2 \pi$ in $t$ and such that

$$
\max _{0 \leqq t \leqq 2 \pi}\left|h_{j}(t)-h_{j}^{(1)}(t)\right|<\epsilon_{0}, \quad(j=1, \cdots, q),
$$

and such that if $h_{j}(t)$ is replaced by $h_{j}^{(1)}(t)$ in the integrals in (6), $j=1, \cdots, q$, the resulting values $b_{1}^{(1)}, \cdots, b_{a}^{(1)}$ are the coordinates of point $p$.

Proof. This follows from inspection of the integrals in (6). For example, we may take:

$$
h_{1}^{(1)}(t)=h_{1}(t)+\frac{a}{\pi} \cos \left(N_{1} t\right)
$$




$$
h_{2}^{(1)}(t)=h_{2}(t)+\frac{b}{\pi} \sin \left(N_{1} t\right),
$$

where $a, b$ are arbitrary small constants.

Definition. Given $\epsilon_{0}>0$, then functions $h_{1}(t), \cdots, h_{q}(t)$ are said to be varied less than $\epsilon_{0}$ if they are replaced by functions $h_{1}^{(1)}(t), \cdots$, $h_{n}^{(1)}(t)$, all of period $2 \pi$ in $t$, and all having continuous second derivatives, and such that

$$
\max _{0 \leqq t \leqq 2 \pi}\left|h_{j}(t)-h_{j}^{(1)}(t)\right|<\epsilon_{0} .
$$

Now we need a lemma about topological degree which was shown in a previous paper [4] to be an easy consequence of a theorem due to Sard [5].

Lemma 4. Let $J$ be a continuous map defined on the closure $\bar{R}$ of a region $R \subset R^{n}$ and differentiable in $R$. Suppose that the topological degree of $J$ at point $p_{0}$ is $d \neq 0$. Then there is a neighborhood $U$ of $p_{0}$ and a set $E$ of $n$-dimensional measure zero, $E \subset U$, such that $p \in U-E$ implies that $J^{-1}(p)$ is a finite set consisting of at least $|d|$ points.

Proof. See [4, Lemma 3.2].

In the two lemmas and the theorem which follow, Lemma 4 will be applied. Functions $h_{1}(t), \cdots, h_{q}(t)$ will be varied less than $\epsilon_{0}$ and in such a way as to avoid a set of zero measure of the kind referred to in Lemma 4. For brevity, we shall refer to this kind of variation of $h_{1}(t), \cdots, h_{q}(t)$ simply as varying $h_{1}(t), \cdots, h_{q}(t)$ less than $\epsilon_{0}$.

Lemma 5. If the topological degree at $O$ of $M$ relative to sphere $S$ is $d \neq 0$, then if $h_{1}(t), \cdots, h_{q}(t)$ are varied less than $\epsilon_{0}$, an arbitrary positive number, then the system

$$
M\left(c_{n-q+1}^{\prime}, \cdots, c_{n}^{\prime}\right)=0
$$

has exactly $m$ solutions inside sphere $S$, where $m \geqq|d|$ and $J(M) \neq 0$ at each solution in $S$ of (3).

Proof. Follows directly from Lemmas 3 and 4.

Lemma 6. If the topological degree of $M$ at $O$ and relative to $S$ is $d \neq 0$, and if $\epsilon_{0}$ is an arbitrary positive number, then there exists $\mu_{1}>0$ such that if $h_{1}(t), \cdots, h_{q}(t)$ are varied less than $\epsilon_{0}$, then for $|\mu|<\mu_{1}$, the system

$$
\mathfrak{M}_{\mu}\left(c_{1}^{\prime}, \cdots, c_{n}^{\prime}\right)=0
$$

has $m$ distinct solutions

$$
\left(c_{1}^{(i)}(\mu), \cdots, c_{n}^{(i)}(\mu)\right), \quad(i=1, \cdots, m) .
$$


Each solution is a continuous function of $\mu$ for $|\mu|<\mu_{1}$. Also $\left(c_{1}^{(i)}(\mu), \cdots, c_{n}^{(i)}(\mu)\right)$ is in the interior of $\mathcal{S}$ for $i=1, \cdots, m$ and for $|\mu|<\mu_{1}$. The solutions are distinct in the following sense: if $\left|\mu_{2}\right|<\mu_{1}$, $\left|\mu_{3}\right|<\mu_{1}$ and if $i_{1} \neq i_{2}$, then

$$
c_{j}^{\left(i_{1}\right)}\left(\mu_{2}\right) \neq c_{j}^{\left(i_{2}\right)}\left(\mu_{3}\right), \quad(j=1, \cdots, n) .
$$

Proof. From Lemmas 1 and 5, it follows that

$$
\mathfrak{T C}_{0}\left(c_{1}^{\prime}, \cdots, c_{n}^{\prime}\right)=0
$$

has $m$ solutions at which $J\left(M_{0}\right) \neq 0$. Also (7) has no other solutions by Lemma 1. The conclusion of the lemma then follows from Lemma 2 because the topological degree is the sum of the signs of the Jacobians. (Each solution $\left(c_{1}^{(i)}(\mu), \cdots, c_{n}^{(i)}(\mu)\right)$ is a continuous function of $\mu$ by the implicit function theorem.)

Theorem. If the topological degree of $M$ at $O$ and relative to $S$ is $d \neq 0$ and if $\epsilon_{0}$ is an arbitrary positive number, then there exists $\mu_{1}>0$ such that if $h_{1}(t), \cdots, h_{q}(t)$ are varied less than $\epsilon_{0}$, equation (E) has $m(\geqq|d|)$ distinct periodic solutions $x_{i}(t, \mu, c)(i=1, \cdots, m)$ where $c$ is in the interior of $\delta$, solution $x_{i}(t, \mu, c)$ depends continuously on $\mu$ for $|\mu|<\mu_{1}$ and

$$
\lim _{\mu \rightarrow 0} x_{i}(t, \mu, c)=e^{t A} c^{(i)}
$$

where $\left(c^{i}\right)=\left(c_{1}^{(i)}(0), \cdots, c_{n}^{(i)}(0)\right)$ for $i=1, \cdots, m$.

Proof. Follows from the derivation of (1), and Lemma 6, and the general existence theorem for solutions of $(\mathrm{E})$.

REMARK. Note that the theorem gives no information about the existence or nonexistence of periodic solutions $x(t, \mu, c)$ such that $c$ is outside $S$. If the radius of $S$ is changed, then, in general, the value of $\mu_{1}$ (see Lemma 6 ) is changed.

REMARK. If (E) is totally degenerate, i.e., if $n=q$, the proof of the theorem goes through in the same way except that Lemma 1 can be omitted and Lemma 2 is simpler.

REMARK. If the topological degree of $M$ at $O$ and relative to $S$ is zero, then the same kind of arguments as have been used here may be applied to show that if equation (3) has solutions in $S$, and if $h_{1}(t), \cdots, h_{q}(t)$ are varied less than $\epsilon_{0}$, then (E) has a finite set of families $x_{i}(t, \mu, c)$ of periodic solutions as described in our theorem. But equation (3) may have no solutions in $S$. If the topological degree is zero, it is generally necessary to employ finer methods to obtain existence theorems (see [3]). 


\section{REFERENCES}

1. E. A. Coddington and N. Levinson, Theory of ordinary differential equations, New York, McGraw-Hill Co., 1955.

2. J. Cronin, Note to Poincarê's perturbation method, Duke Math. J. vol. 26 (1959) pp. 251-262.

3. - Poincare's perturbation method and topological degree, to appear in Contributions to Nonlinear Differential Equations, vol. 5, Princeton University Press.

4. - Branch points of solutions of equations in Banach space. II, Trans. Amer. Math. Soc. vol. 76 (1954) pp. 207-222.

5. A. Sard, The measure of the critical values of differentiable maps, Bull. Amer. Math. Soc. vol. 48 (1942) pp. 883-890.

Polytechnic Institute of Brooklyn

\section{QUOTIENT GROUPS OF REDUCED ABELIAN GROUPS}

\section{ELBERT A. WALKER}

Let $G$ be a reduced torsion $p$-group. (In this paper, group will mean Abelian group.) Let $B$ be a basic subgroup of $G$. It is well known that $|B| \wedge_{0} \geqq|G|$, where $|S|$ denotes the cardinal of the set $S$. Fuchs gives a proof of this in $[1$, p. 102], and attributes it to Kulikov. This has turned out to be a very useful fact, and the purpose of this short note is to generalize it. Now, as is generally known, every torsion group $G$ has a basic subgroup $B$; that is, a pure subgroup $B$ that is a direct sum of cyclic groups, and such that $G / B$ is divisible. To obtain such a $B$, simply take $B_{p}$ to be a basic subgroup of the $p$-component of $G$, and let $B=\sum_{p} \oplus B_{p}$. It is easy to see that in this more general situation $|B| \aleph_{0} \geqq|G|$ still holds, and in fact follows from the corresponding statement for $p$-groups. The generalization we will prove is the following

THEOREM. Let $G$ be a reduced torsion group, and let $H$ be a subgroup of $G$ such that $G / H$ is divisible. Then $|H| \aleph_{0} \geqq|G|$.

Proof. Our proof uses some homological results of Harrison in [2]. Notice that we may assume that $|H|<|G|$, that $G$ is infinite, and hence that $|G / H|=|G|$. Let $Q$ and $Z$ denote the additive group of rationals and integers, respectively. From the exact sequence

$$
0 \rightarrow H \rightarrow G \rightarrow G / H \rightarrow 0,
$$

we get the exact sequence

Received by the editors March 17, 1960. 